\title{
Spreading of Micrometer-Sized Droplets under the Influence of Insoluble and Soluble Surfactants: A Numerical Study
}

\author{
Thomas Antritter ${ }^{1, *} \mathbb{D}$, Peter Hachmann ${ }^{2}$, Tatiana Gambaryan-Roisman ${ }^{1} \mathbb{D}$, Bernhard Buck ${ }^{2}$ \\ and Peter Stephan ${ }^{1}$ \\ 1 Institute for Technical Thermodynamics, Technische Universität Darmstadt, Alarich-Weiss-Straße 10, \\ 64287 Darmstadt, Germany \\ 2 Heidelberger Druckmaschinen AG, Gutenbergring, 69168 Wiesloch, Germany \\ * Correspondence: antritter@ttd.tu-darmstadt.de
}

Received: 12 July 2019; Accepted: 2 August 2019; Published: 9 August 2019

\begin{abstract}
Wetting and spreading of surfactant solutions play an important role in many technical applications. In printing processes, the size of individual droplets is typically on the order of a few tens of microns. The purpose of this study is to develop a better understanding of the interaction between spreading and surfactant transport on these small length and related time scales. Therefore, numerical simulations based on the volume-of-fluid method including Marangoni stresses and transport of an insoluble or soluble surfactant are performed. The results for an insoluble surfactant show competing effects of Marangoni flow on the one hand, and a decreasing surfactant concentration as the droplet spreads on the other hand. Even in the case of a soluble surfactant, adsorption and desorption could only partly mitigate these effects, demonstrating the importance of the sorption kinetics for fast, small scale wetting processes.
\end{abstract}

Keywords: wetting; spreading; complex fluids; surfactant; computational fluid dynamics; volume-of-fluid method

\section{Introduction}

Spreading of micrometer-sized droplets plays an important role in various technical applications, especially in printing processes. In inkjet printing, for example, the interaction between droplets and the substrate has an important influence on resolution and print quality [1], as this interaction determines dot size and shape. Continuous features are formed by consecutively placed droplets. Therefore, the spreading of single droplets determines the initial state before coalescence or collision at the substrate. Especially on impermeable substrates, coalescence can cause the so-called 'mottling' printing defect [2]. Understanding the spreading of single ink droplets is consequently an important step towards systematic optimization of the printing process. For pure Newtonian liquids on flat, impermeable substrates wetting is fairly well understood. A review can be found in [3,4]. However, typical inks show a more complex behavior due to the presence of the pigments and surface active substances, so-called surfactants. Harikrishnan et al. [5] studied the spreading of sessile droplets under the influence of nanoparticles and surfactants, focusing on the interaction between surfactants and nanoparticles. For both nanocolloidal systems with and without surfactant present, they found a deviation from Tanner's Law [6] $d \propto t^{n}$, where $d$ is the wetted diameter and $t$ stands for time. They observed significantly smaller $n$ than the expected value of 0.1 for pure liquids and concluded that surfactant effects dominated the spreading dynamics when surface active substances were present. Kovalchuk et al. [7] experimentally investigated surfactant enhanced spreading for two surfactants with different adsorption kinetics. They found that a slower surfactant supply from the bulk towards 
the leading edge leads to a Marangoni flow towards the three phase contact line, resulting in faster spreading. In their case, the surfactant showed the opposite influence on wetting compared to [5].

Wetting under the influence of surfactants has been investigated theoretically and numerically before. To study the effect of solutal Marangoni stresses due to an insoluble surfactant on spreading, lubrication theory has been applied to the contact line region [8,9] or sessile droplets [10-12]. Karapetsas et al. [13] included the effect of solubility as well as surfactant transfer from the liquid-air interface to the substrate and concluded that the latter plays a crucial role in superspreading. The lubrication approximation, however, limits the above investigations to the thin film limit. Numerical methods for two-phase flows such as the phase-field, level-set or the volume-of-fluid method allow the simulation of wetting phenomena beyond the thin film limit and generally also allow for topological changes e.g., during droplet impingement, coalescence or collision. The effect of surfactant transport and corresponding surface tension variation has previously been included into these methods. Teigen et al. [14] introduced a method for taking into account soluble surfactants in the diffuse interface context and applied it to various test cases, however not to wetting scenarios. Lakshmanan and Ehrhard [15] present a method for the description of insoluble surfactants based on the level-set method and employ it to study the effect of surfactants on the rise of gas bubbles. For the geometric volume-of-fluid method, numerical methods for the description of soluble [16] and insoluble [17] surfactants have been presented. However, none of the above considered wetting under the influence of soluble surfactants on the micrometer length scale.

In the present work, we investigate the influence of insoluble and soluble surfactants on the spreading of micrometer-sized droplets. Due to their small size, the ratio of interfacial area to the droplet volume is significantly smaller compared to the typically observed millimeter-sized droplets. In addition, the capillary time scale for the hydrodynamics of wetting $t^{*}=\frac{d \mu}{\sigma}$ with the dynamic viscosity $\mu$ and the surface tension $\sigma$ is smaller in the case of micrometer-sized droplets leading to a higher expansion rate of the interfacial area. We therefore focus on the competing effects of spreading and surfactant adsorption at the liquid-gas interface while taking the solutal Marangoni effect into account. A model for soluble surfactants was integrated into the algebraic volume-of-fluid method based solver InterFoam from the open source CFD library OpenFOAM [18] extended by additional functionality provided by the interface and contact line library (ICLL) first employed in [19] and described in more detail in [20].

In the following section, we will first present the governing equations and the numerical method. Following that, we show verification cases for the sorption model as well as the Marangoni stresses at the beginning of Section 3. Afterwards, simulation results for spreading droplets with insoluble and soluble surfactants will be presented, followed by the discussion of the results.

\section{Method}

In this section, we present the governing equations for the two-phase flow taking into account surface tension forces, surfactant transport, and the model for the three phase contact line, followed by a brief description of the numerical procedure. Afterwards, we present the considered parameter range and model setup for the results shown in Section 3.

\subsection{Model Equations}

Within the algebraic volume-of-fluid method, the two-phase flow is described by the volume averaged conservation equations for mass and momentum. The mass balance for two incompressible, immiscible fluids without slip between the two phases and without phase change then takes the form

$$
\begin{gathered}
\frac{\partial \alpha}{\partial t}+\nabla \cdot \alpha \mathbf{u}+\nabla \cdot\left(\alpha(1-\alpha) \mathbf{u}_{\mathrm{r}}\right)=0, \\
\nabla \cdot \mathbf{u}=0,
\end{gathered}
$$


where in our case $\alpha$ represents the volume fraction of the liquid phase and $\mathbf{u}$ is the mean velocity of the two phases. Furthermore, the InterFoam solver employs an artificial compressive velocity $\mathbf{u}_{\mathrm{r}}=c_{\mathrm{r}}|\mathbf{u}| \mathbf{n}$ in order to counteract numerical diffusion, where the interface normal vector is evaluated from $\mathbf{n}=\frac{\nabla \alpha}{|\nabla \alpha|}$ and $c_{\mathrm{r}}=1$ in the present study. The momentum balance is given by

$$
\frac{\partial \rho_{\mathrm{m}} \mathbf{u}}{\partial t}+\nabla \cdot\left(\rho_{\mathrm{m}} \mathbf{u u}\right)=\nabla \cdot\left[\mu_{\mathrm{m}}\left(\nabla \mathbf{u}+(\nabla \mathbf{u})^{\mathrm{T}}\right)\right]-\nabla p+\rho_{\mathrm{m}} \mathbf{g}+\mathbf{f}_{\mathrm{c}}
$$

where $\rho_{\mathrm{m}}=\alpha \rho_{\ell}+(1-\alpha) \rho_{\mathrm{g}}$ and $\mu_{\mathrm{m}}=\alpha \mu_{\ell}+(1-\alpha) \mu_{\mathrm{g}}$ represent the averaged density and dynamic viscosity of liquid $(\ell)$ and gas phase $(\mathrm{g})$, respectively, and $p$ is the pressure. The capillary forces include Marangoni stresses and are modeled as a body force through the continuum surface force model (CSF) [21] by

$$
\mathbf{f}_{\mathrm{c}}=-\sigma \kappa \delta_{\mathrm{f}} \mathbf{n}+\delta_{\mathrm{f}} \nabla_{\Sigma} \sigma,
$$

with the surface tension $\sigma$ and the curvature $\kappa=\nabla \cdot \mathbf{n} . \nabla_{\Sigma}=(\mathbb{I}-\mathbf{n n}) \nabla$ represents the interface tangential component of the gradient. Transferring the density-scaled CSF model [22] to the volume-of-fluid method, $\delta_{\mathrm{f}}=2 \alpha|\nabla \alpha|$. Assuming that the surfactant is soluble only in the liquid phase, mass conservation in the bulk phase can be described by

$$
\frac{\partial c_{\mathrm{B}}}{\partial t}+\nabla \cdot\left(c_{\mathrm{B}} \mathbf{u}\right)+\nabla \cdot\left(c_{\mathrm{B}}(1-\alpha) \mathbf{u}_{\mathrm{r}}\right)=\nabla \cdot\left(D_{\mathrm{B}} \nabla c_{\mathrm{B}}\right)-\nabla \cdot\left(\frac{c_{\mathrm{B}}}{\alpha} D_{\mathrm{B}} \nabla \alpha\right)-j_{\mathrm{B}},
$$

where we introduced an artificial compression term similar to the term in the advection equation for the phase fraction counteracting numerical diffusion of the surfactant into the gas phase. However, note that this term is non-zero only in the interface region, where $c_{\mathrm{B}}>0$ and $\alpha<1$. $c_{\mathrm{B}}$ stands for the surfactant concentration in the liquid phase, $j_{\mathrm{B}}$ is a sink term due to adsorption of surfactant at the interface and $D_{\mathrm{B}}$ is the diffusion coefficient for the bulk phase. The diffusive term on the right-hand side is a simplification of the term given by Deising et al. [23] for the limiting case of solubility of the solute in only one of the two bulk phases. Following Lakshmanan and Ehrhard [15], we also model the surfactant in the interface region as a volume specific concentration $c_{\mathrm{I}}$. The species transport at the interface is then described by

$$
\frac{\partial c_{\mathrm{I}}}{\partial t}+\nabla \cdot\left(c_{\mathrm{I}} \mathbf{u}\right)+\nabla \cdot\left(c_{\mathrm{I}}(1-2 \alpha) \mathbf{u}_{\mathrm{r}}\right)=\nabla_{\Sigma} \cdot\left(D_{\Gamma} \nabla_{\Sigma} c_{\mathrm{I}}\right)+j_{\mathrm{I}}
$$

where $j_{\mathrm{I}}$ denotes a source term due to adsorption from the bulk and $D_{\Gamma}$ is the diffusion coefficient along the interface. Similar to [15], a convective compression term is added to confine the surfactant to the interface region. Note that this term is zero at the liquid-gas interface (i.e., for $\alpha=0.5$ ) and pointed towards the interface everywhere else. The two fields for surfactant transport in the bulk phase and at the interface are coupled through a Langmuir-Hinshelwood kinetic model. With an approach similar to the continuum surface force model, the source terms for the surfactant concentration at the interface due to adsorption are calculated as

$$
j_{\mathrm{I}}=k_{\mathrm{ad}} \mathcal{C}_{\mathrm{B} \rightarrow \mathrm{I}}\left(\delta_{\mathrm{c}}-\frac{c_{\mathrm{I}}}{\Gamma_{\mathrm{m}}}\right)-k_{\mathrm{de}} \mathcal{C}_{\mathrm{I}}
$$

where $\delta_{\mathrm{c}}=|\nabla \alpha|, k_{\mathrm{ad}}$ and $k_{\mathrm{de}}$ are adsorption and desorption rate coefficients and $\Gamma_{\mathrm{m}}$ is the (area specific) maximum surface concentration of surfactant. The sub-surface bulk concentration $c_{B \rightarrow I}$ is evaluated at a distance of approximately two mesh widths from the interface. The source or sink terms for the bulk concentration $j_{\mathrm{B}}$ are then evaluated by distributing $j_{\mathrm{I}}$ into the liquid phase by solving an inhomogeneous Helmholtz equation for $j_{\mathrm{I}}$, cutting off the smoothed source term field in the interface region and the gas phase and rescaling the remaining source terms to ensure global mass conservation. This method approximates the local distribution of source terms, however, in general violates local mass conservation. It is similar to the procedure for source and sink terms for mass and energy balance 
equations due to evaporation proposed by Hardt and Wondra [24]. Analogous to their evaporation model, the smoothed source term field should only extend over a few grid cells necessary to avoid numerical instabilities. Therefore, with increasing mesh, resolution errors in local mass conservation are expected to decrease.

In order to be able to apply common surface equations of state, relating the surface tension to the (area specific) surfactant concentration at the interface $\Gamma$, this concentration is evaluated in the interface region from

$$
\Gamma=\frac{c_{\mathrm{I}, \mathrm{s}}}{\delta_{\mathrm{c}, \mathrm{s}}}
$$

where the smoothed concentration and interface density fields $c_{I, s}$ and $\delta_{\mathrm{c}, \mathrm{s}}$ are obtained from the solution of inhomogeneous Helmholtz equations. The local surface tension is then in the present work calculated from the Frumkin equation of state [25]

$$
\sigma=\sigma_{0}+R T \Gamma_{\mathrm{m}} \ln \left(1-\frac{\Gamma}{\Gamma_{\mathrm{m}}}\right)
$$

with the surface tension of the pure solvent $\sigma_{0}$, the universal gas constant $R$ and the temperature $T$. The moving contact line is treated by including the Kistler model [26]

$$
\theta_{\mathrm{d}}=f_{\text {Hoff }}\left[C a+f_{\text {Hoff }}^{-1}\left(\theta_{\mathrm{e}}\right)\right]
$$

with the Hoffman function

$$
f_{\text {Hoff }}(x)=\cos ^{-1}\left\{1-2 \tanh \left[5.16\left(\frac{x}{1+1.31 x^{0.99}}\right)^{0.706}\right]\right\}
$$

as a subgrid model for the dynamic contact angle along the substrate, where the capillary number $C a=u_{\mathrm{cl}} \mu / \sigma$ is evaluated using the local surface tension. The contact line velocity $u_{\mathrm{cl}}$ is calculated from the bulk velocity field in the vicinity of the contact line using the procedure presented in [27].

\subsection{Numerical Method}

Within each time step, first the advection equation for the phase fraction, Equation (1), is solved. Following this, the surfactant transport Equations (5)-(7) are solved. We iterate over these until the coupling through the source terms is converged. Afterwards, the interface normal vectors along the substrate are corrected according to Equation (10) and the surface tension forces are calculated. Finally, the pressure-velocity coupling is solved using the PISO-Algorithm [28]. The time step is dynamically adjusted according to the time step criterion

$$
\Delta t_{\mathrm{DAT}} \leq \max \left(10 \frac{\mu h}{\sigma}, 0.1 \sqrt{\frac{\rho h^{3}}{\sigma}}\right)
$$

suggested for the InterFoam solver by Deshpande et al. [29], where, in the present simulation, the criterion is evaluated in the vicinity of the liquid-gas interface with $\rho_{\mathrm{m}}$ and $\mu_{\mathrm{m}}$.

\subsection{Parameters for Droplet Spreading}

The parameters in the present study are based on typical values in inkjet printing with UV-curable inks. Droplet diameters in inkjet printing are typically in the range of tens of microns [30]. Here, we considered droplets with a volume of $V=6 \mathrm{pL}$, corresponding to a diameter of $22.55 \mu \mathrm{m}$. The main solvent in these inks are typically mono- or diacrylate monomers [31], the density of which is approximately $1000 \mathrm{~kg} \mathrm{~m}^{-3}$. A usual range of the viscosity of inkjet inks is 2 to $50 \mathrm{mPa}$ [ [30]. Therefore, we consider here $\mu_{\ell}=20 \mathrm{mPa}$ s as an intermediate value within that range. For the gas 
phase, we assume $\rho_{\mathrm{g}}=1.2 \mathrm{~kg} \mathrm{~m}^{-3}$ and $\mu_{\mathrm{g}}=19 \mu \mathrm{Pa}$ s. The surface tension of UV-curable inkjet inks without surfactant is $\sigma_{0} \approx 35 \mathrm{mN} \mathrm{m}^{-1}$. Only limited data regarding the sorption kinetics for typical surfactants in these systems are available. Bale [32] presented dynamic surface tension measurements using maximum bubble pressure tensiometry of UV-inks with different silicone additives. These measurements show a decrease of the surface tension starting on a timescale of $1 \mathrm{~ms}$ and it takes the system several seconds to minutes to reach a stationary equilibrium surface tension $\sigma_{\mathrm{eq}} \approx 23 \mathrm{mN} \mathrm{m}^{-1}$. Since we are mainly interested in the qualitative spreading behavior, we assumed $k_{\mathrm{ad}}=6 \times 10^{-4} \mathrm{~m} \mathrm{~s}^{-1}$, $k_{\mathrm{de}}=1.1 \times 10^{2} \mathrm{~s}^{-1}, \Gamma_{\mathrm{m}}=6 \times 10^{-5} \mathrm{~mol} \mathrm{~m}^{-2}$ [33] and $D_{\mathrm{B}}=5.3 \times 10^{-10} \mathrm{~m}^{2} \mathrm{~s}^{-1}$ [34] corresponding to heptanol in water and adjusted $\sigma_{0}=35 \mathrm{mN} \mathrm{m}^{-1}$ as well as the initial surfactant concentrations to fit the initial and equilibrium values of UV-curable ink given by Bale [32]. Even though this can only be a rough approximation, this system shows a dynamic surface tension behavior similar to typical UV-curable inkjet inks. Figure 1 shows the dynamic surface tension for the chosen model parameters over the surface age for an initially surfactant free, planar interface. The surface tension was evaluated from the surface concentration data obtained with our numerical model and presented in Section 3.1 using the Frumkin equation of state (Equation (9)). Concentration and surface tension data as well as further data presented throughout this article are also available as electronic Supplementary Material. With the chosen model parameters and the initial surfactant concentration $c_{\infty}=0.941 \mathrm{~mol} \mathrm{~m}^{-3}$ in the liquid phase, the system shows a similar behaviour compared to the measurements presented by [32].

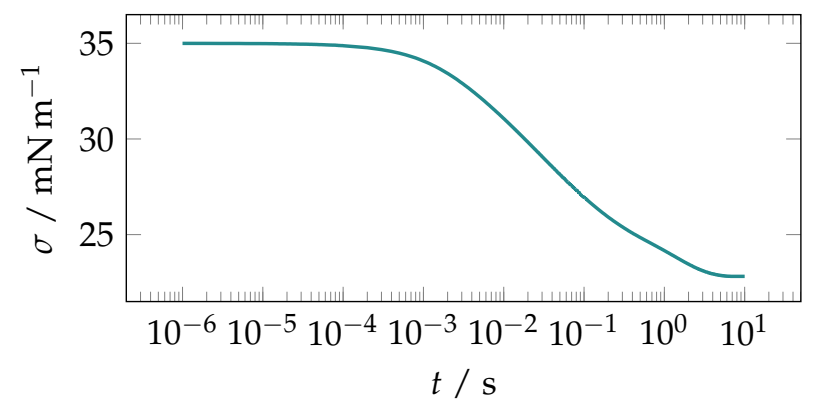

Figure 1. Dynamic surface tension over surface age with the model parameters presented in Section 2.3.

\subsection{Numerical Setup for Droplet Spreading}

For the simulations presented in Sections 3.3 and 3.4, the droplet is initialized as a hemisphere with a volume of $6 \mathrm{pL}$ and is initially at rest. We perform $2 \mathrm{D}$ axisymmetric simulations taking advantage of the rotational symmetry of the problem. Figure 2 shows a sketch of the computational domain including the initial conditions for the volume fraction $\alpha$, bulk and interface concentrations $c_{\mathrm{B}}$ and $c_{\mathrm{I}}$, and the pressure field with $p_{\mathrm{YL}}=2 \sigma / R$, where $R=14.20 \mu \mathrm{m}$ is the initial curvature radius. A no slip and no penetration condition was used and the normal pressure gradient was set to zero along the substrate. For the inlet/outlet boundaries at the top and side of the computational domain, a zero gradient condition for the velocity field and a fixed value of $p=0$ for the pressure were set. Depending on the flow direction, the boundary condition for the volume fraction as well as for concentrations $c_{\mathrm{B}}$ and $c_{\mathrm{I}}$ changes between a fixed value of zero for an inflow and zero gradient condition for an outflow. The domain is resolved with $105 \times 120$ cells in axial and radial directions. Grading was used to increase the mesh resolution towards the axis and towards the substrate resulting in mesh sizes between $0.09 \mu \mathrm{m}$ and $0.47 \mu \mathrm{m}$. The setup is therefore similar to previous work [27], except the fact that, in the present case, it additionally includes initial conditions for the surface active substance. 


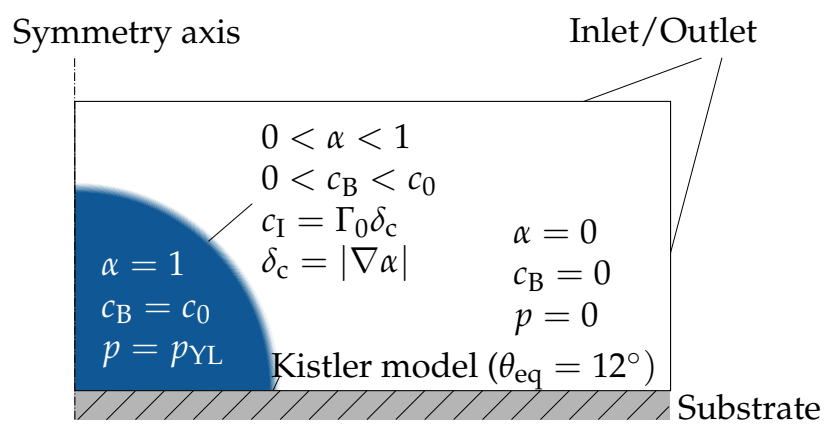

Figure 2. Domain with initial and boundary conditions for droplet spreading simulations.

\section{Results}

Before presenting the results for spreading droplets, we first show results of two verification cases for (i) the sorption model in Section 3.1 and (ii) the implementation of Marangoni stresses in Section 3.2. Following that, the results for spreading $\mu \mathrm{m}$-sized droplets with insoluble (Section 3.3) and soluble (Section 3.4) surfactant are presented.

\subsection{Verification of the Sorption Model}

One of the main novelties compared to the convective-diffusive species transport models at the interface and within the bulk, given in [15,23], respectively, is the coupling of the two fields through a sorption model. In order to verify our implementation of the said sorption model, we considered the one-dimensional problem of sorption to a planar interface from the bulk of a liquid. The initial surfactant concentration within the bulk is assumed to have a constant value of $c_{\infty}=0.941 \mathrm{~mol} \mathrm{~m}^{-3}$ while the interface is initially free of surfactant $(\Gamma=0)$. As a reference, we used the two limiting cases for the mixed kinetic model of (i) diffusion controlled adsorption and (ii) Langmuir-Hinshelwood kinetics. For the former case, the concentration at the interface can be described by the Ward-Tordai equation

$$
\Gamma(t)=2 \sqrt{\frac{D}{\pi}} c_{\infty}\left(\sqrt{t}-\left.\int_{0}^{\sqrt{t}} c\right|_{x=0}(\tau) \mathrm{d} \sqrt{t-\tau}\right),
$$

where $\left.c\right|_{x=0}$ is the subsurface bulk concentration [35]. In order to verify our implementation, we integrated this equation numerically using the $\mathrm{C}++$ code provided by Li et al. [36]. For the latter case, where the sorption kinetics pose the rate determining step, the sorption process can be described by the Langmuir-Hinshelwood kinetic model

$$
\dot{\Gamma}(t)=\left.k_{\mathrm{a}} c\right|_{x=0}-\left(\frac{\left.k_{\mathrm{a}} c\right|_{x=0}}{\Gamma_{\mathrm{m}}}+k_{\mathrm{d}}\right) \Gamma
$$

with a constant subsurface concentration of $\left.c\right|_{x=0}=c_{\infty}$. The surfactant concentration at the interface is then given by solution of this ordinary differential equation

$$
\Gamma(t)=\frac{\Gamma_{\mathrm{m}} k_{\mathrm{a}} c_{\infty}}{k_{\mathrm{a}} c_{\infty}+\Gamma_{\mathrm{m}} k_{\mathrm{d}}}\left\{1-\exp \left[-\left(\frac{k_{\mathrm{a}} c_{\infty}}{\Gamma_{\mathrm{m}}}+k_{\mathrm{d}}\right) t\right]\right\} .
$$

For both reference cases, we used the material properties of heptanol in water from the previous section. Dimensional analysis of the one-dimensional planar adsorption problem leads to two dimensionless parameters. Similar to Ferri and Stebe [37], we characterize the equilibrium state through the dimensionless number $\Pi_{1}=\frac{k_{\mathrm{a}} c_{\infty}}{k_{\mathrm{d}} \Gamma_{\mathrm{m}}}$. With the material properties and bulk concentration given above, this leads to $\Pi_{1} \approx 8.55 \times 10^{-2}$. In addition to that, the ratio of kinetic sorption rate to diffusive transport is described by $\Pi_{2}=\frac{k_{\mathrm{a}}^{2}}{D k_{\mathrm{d}}}$. For heptanol in water, $\Pi_{2}=1.62 \times 10^{-1}$. To compare against the two limiting cases described, we additionally considered $\Pi_{2}=10^{-2}$ and $\Pi_{2}=10^{2}$. The numerical results from the mixed kinetics model were obtained on a one-dimensional mesh with a 
resolution of $\Delta x=1.875 \times 10^{-7} \mathrm{~m}$. To resolve the concentration gradients at the interface in the early stages of the diffusion controlled case $\left(\Pi_{2}=10^{2}\right)$, the mesh was locally refined down to a mesh size of $2.930 \times 10^{-9} \mathrm{~m}$. The domain size is $15 h$ with $h=\frac{\Gamma}{c_{\infty}}$ being the characteristic length scale of the diffusion controlled adsorption process [37]. The interface position is, just as for the two-dimensional cases considered in the following sections, implicitly given by the volume fraction of the liquid phase $\alpha$. It is placed at $10 h$ from the boundary, leaving $2 / 3$ of the domain for the diffusion process. As the two reference cases consider only diffusive transport and adsorption, respectively, the fluid is assumed to be at rest. The solution of the momentum balance as well as the advection equation of the phase fraction $\alpha$ is therefore not necessary in this case. The results are consequently obtained from the solution of Equations (5)-(8).

Figure 3 shows the results obtained with our numerical model for varying $\Pi_{2}$ in comparison with the reference solutions. For the parameters considered here, the reference solutions indicate a transition from the adsorption kinetics limited regime to the diffusion controlled regime at approximately $1 \mathrm{~ms}$. This transition is also captured by our numerical results with the mixed kinetics model for $\Pi_{2}=1.62 \times 10^{-1}$. Furthermore, with an increased diffusion coefficient $\left(\Pi_{2}=10^{-2}\right)$, the results from the numerical model show excellent agreement with the analytical solution given by Equation (15). In the opposite case of increased adsorption and desorption rate coefficient $\left(\Pi_{2}=10^{2}\right)$, excellent agreement with the reference solution of Equation (13) was found.

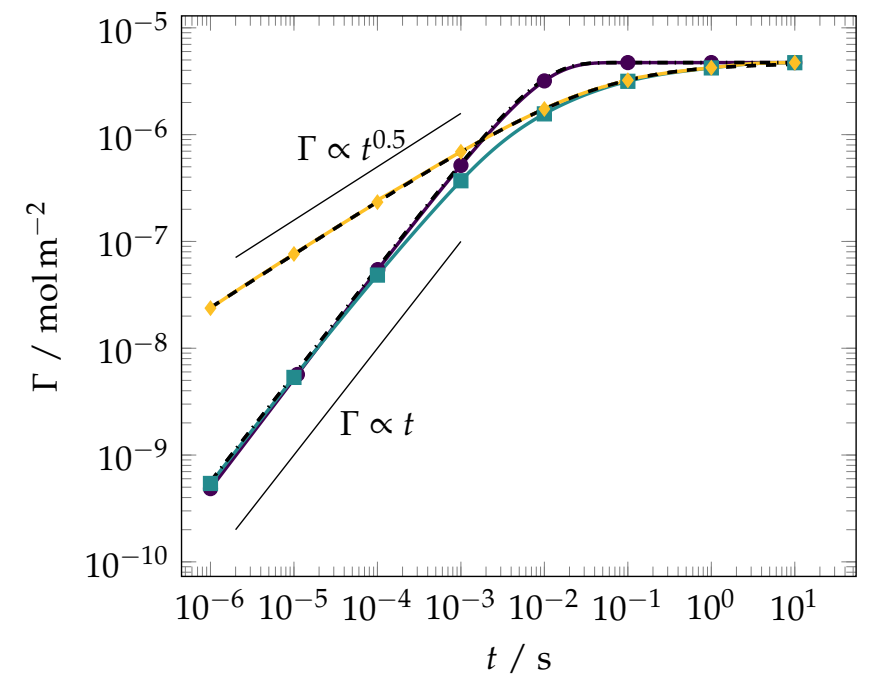

$$
\begin{aligned}
& \text { - - - Ward-Tordai solution } \\
& \text { - - - Langmuir-Hinshelwood kinetics } \\
& \rightarrow-\text { Mixed kinetics model }\left(\Pi_{2}=1 \times 10^{-2}\right) \\
& \rightarrow \text { - Mixed kinetics model }\left(\Pi_{2}=1.62 \times 10^{-1} ; \text { Heptanol }\right) \\
& \rightarrow-\text { Mixed kinetics model }\left(\Pi_{2}=1 \times 10^{2}\right)
\end{aligned}
$$

Figure 3. Verification of the mixed kinetics model with numerical and analytical reference solutions.

\subsection{Verification of the Marangoni Stress Model}

To verify our implementation of the Marangoni stresses, we applied our code to a viscous drop with a fixed surfactant distribution along the interface. This test case has previously been used for the validation of solutal Marangoni stresses in the diffuse interface context by Teigen et al. [14]. The drop is initialised as a sphere on an axisymmetric grid. The droplet is kept in its initial shape, i.e., the transport equation of the phase fraction field Equation (1) (as well as Equations (5)-(7) for surfactant transport) are not solved in this test case. The solution is therefore obtained from Equations (2)-(4) and (8). Instead of Equation (9), a linear surface tension model was employed for this test case. For a linear 
increase in surface tension along the rotational axis, a reference solution for the steady state velocity was given by Young et al. [38]. The surfactant concentration at the interface $c_{\mathrm{I}}$ was set accordingly. Both the droplet and the surrounding continuous phase are initially at rest. Figure 4 shows the mean velocity inside the droplet normalised with the expected value from analytical solution from [38] over the pseudo time $\tau$. Even though the terminal velocity is slightly underpredicted, good agreement with the analytical solution was found.

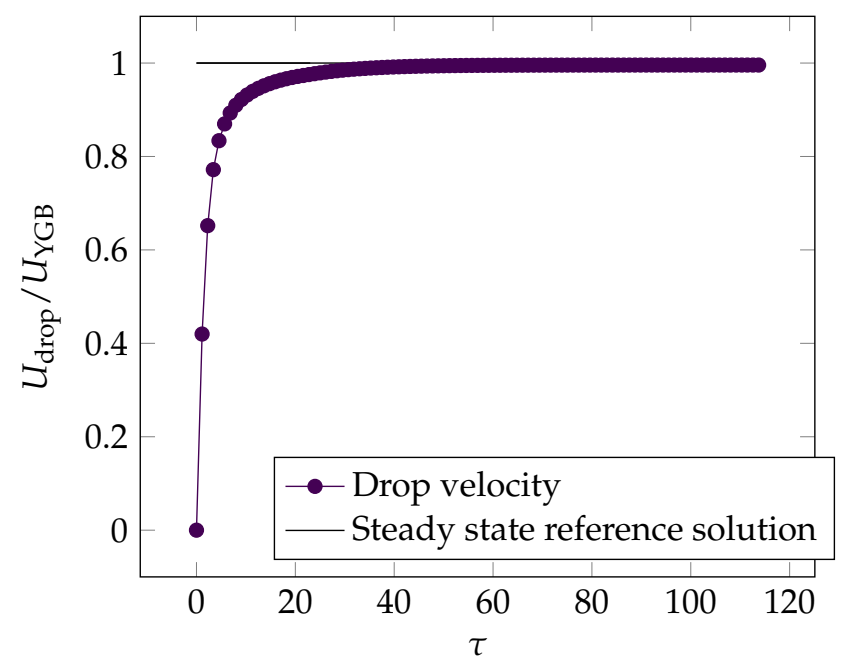

Figure 4. Drop velocity in a linear surfactant concentration gradient in comparison with the theoretical steady state solution from [38].

\subsection{Spreading of Droplet with Insoluble Surfactant}

Consider a spreading droplet with an insoluble surfactant adsorbed at the liquid-gas interface. The fluid properties as well as $\Gamma_{\mathrm{m}}$ are chosen as described in Section 2.3. The sorption parameters $k_{\mathrm{ad}}$ and $k_{\mathrm{de}}$ are set to zero. The droplet is initially at rest. Boundary and initial conditions are set as described in Section 2.4. The initial surfactant concentration at the interface of the hemispherical droplet $\Gamma_{0}=4.727 \times 10^{-6} \mathrm{~mol} \mathrm{~m}^{-2}$ corresponds to a surface tension of $23 \mathrm{mN} \mathrm{m}^{-1}$.

Figure 5 shows the wetted diameter over time for a droplet with insoluble surfactant in comparison with a spreading droplet with the same initial, yet constant surface tension throughout the simulation. Starting from the same initial diameter, the droplet with insoluble surfactant spreads initially slower compared to the droplet with constant surface tension. After approximately $0.1 \mathrm{~ms}$, this dynamics changes, and now the droplet with a constant surface tension of $23 \mathrm{mN} \mathrm{m}^{-1}$ spreads slower compared to the droplet with insoluble surfactant. This results in both droplets reaching the same wetted diameter after a few milliseconds. From then on, the droplet with insoluble surfactant continues to spread slightly faster than its counterpart with constant surface tension and, by the end of the simulation after $10 \mathrm{~ms}$, the droplet with insoluble surfactant has reached a slightly larger wetted diameter. At this stage both droplets have not yet reached their equilibrium diameter corresponding to a contact angle of $12^{\circ}$.

Figure 6 shows the velocity fields for both droplets after $0.1 \mathrm{~ms}$ of spreading, i.e., towards the end of the first stage, where the droplet with insoluble surfactant shows slower wetting compared to the droplet with constant surface tension. The substrate as well as the droplet contour are displayed as black solid lines. For the droplet with a constant surface tension of $23 \mathrm{mN} \mathrm{m}^{-1}$, the largest velocities appear at the liquid-gas interface and are directed towards the three-phase contact line. As could be expected, due to the no slip condition at the substrate, the velocity decreases towards the droplet base. The liquid is transported to the contact line mainly along the liquid-gas interface. The velocity field for the droplet with insoluble surfactant differs from that in several aspects. First of all, the maximum velocity is clearly smaller compared to the droplet with constant surface tension. Furthermore, the flow 
towards the contact line now occurs mainly in the bulk of the droplet. Close to the position where, in the previous case, the maximal velocity was found, now the smallest velocity along the interface can be observed. Clearly, the Marangoni effect plays an important role in this behavior. The velocity field at the liquid-gas interface during the spreading phase is directed to the contact line. The flow leads to the transport of the insoluble surfactant located at the liquid-gas interface towards the contact line. The surface tension at the contact line decreases, inducing Marangoni flow in the opposite direction towards the droplets' center. This limits the transport of surfactant towards the contact line and the corresponding surface tension gradients, resulting in the flow profile shown in Figure $6 \mathrm{~b}$.

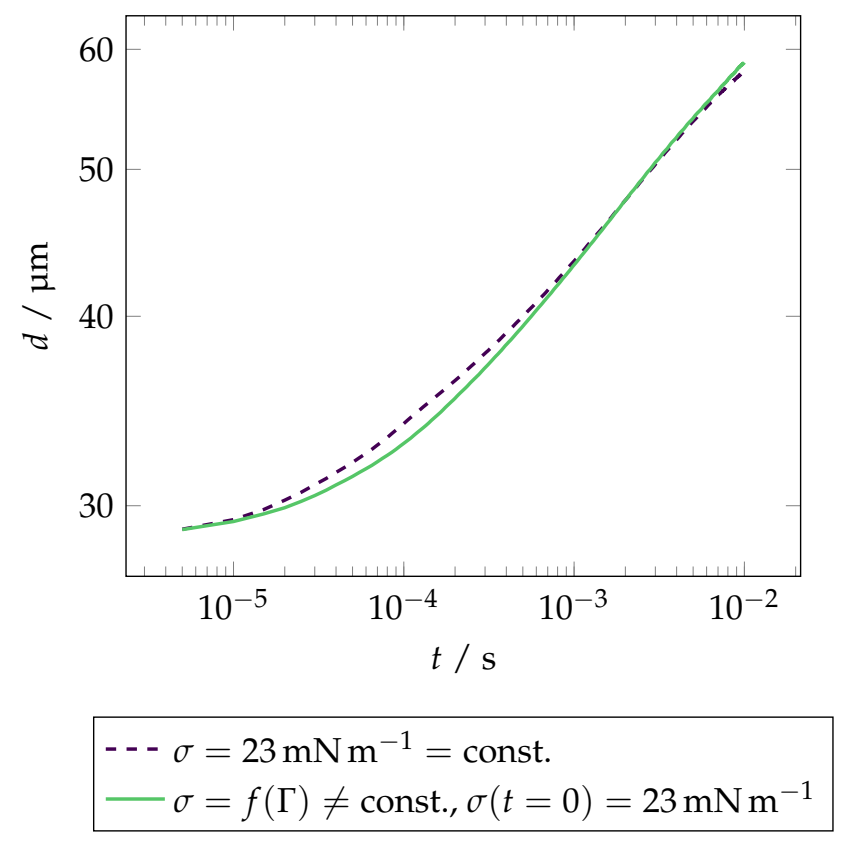

Figure 5. Wetted diameter over time for a droplet with insoluble surfactant in comparison with a droplet with constant, but initially identical surface tension.

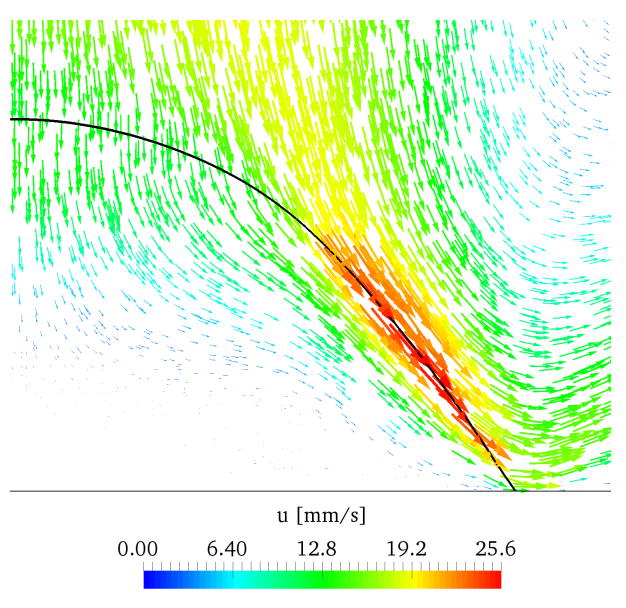

(a) $\sigma=23 \mathrm{mN} \mathrm{m}^{-1}=$ const.

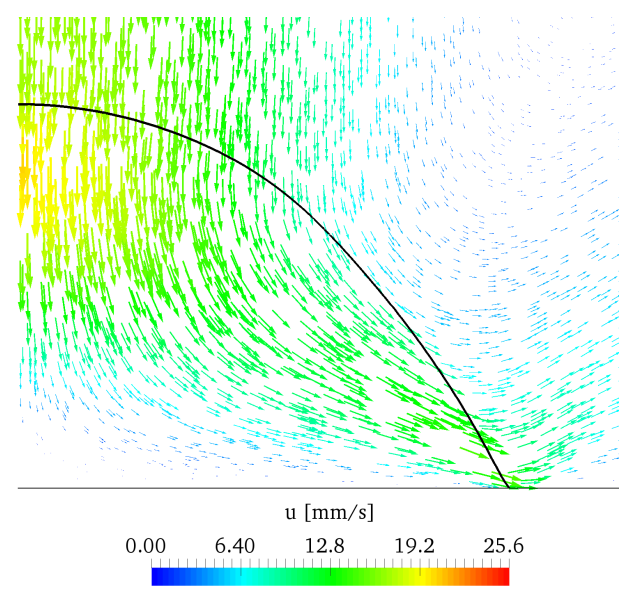

(b) $\sigma=f(\Gamma) \neq$ const., with $\sigma(t=0)=23 \mathrm{mN} \mathrm{m}^{-1}$

Figure 6. Velocity field in droplet and surrounding gas phase after $0.1 \mathrm{~ms}$ for different surface tension models.

The mean surfactant concentration at the liquid-gas interface and the corresponding mean surface tension are depicted in Figure 7 for the droplet with insoluble surfactant. Due to the increasing 
interfacial area during droplet spreading a decreasing surfactant concentration is expected. During the initial spreading stage until $t \approx 0.1 \mathrm{~ms}$, only a slight decrease of the mean surfactant concentration can be observed. Accordingly, also the mean surface tension increases only marginally and remains below $24 \mathrm{mN} \mathrm{m}^{-1}$. During the following spreading, the concentration of the insoluble surfactant clearly decreases and after $10 \mathrm{~ms}$ has more than halved. The mean surface tension increases up to about $30 \mathrm{mN} \mathrm{m}^{-1}$ during that period. This offers an explanation for the increased contact line velocity for the droplet with insoluble surfactant compared to the droplet with constant surface tension in that stage. For a given (dynamic) contact angle, an increased surface tension leads to a larger contact line velocity, as predicted by various contact angle models including the Kistler dynamic contact angle given by Equation (10). The increase in interfacial area and surface tension can therefore be an explanation for the increased spreading rate for the droplet with insoluble surfactant compared to the droplet with constant surface tension observed in Figure 5 in the later stage of the spreading process.

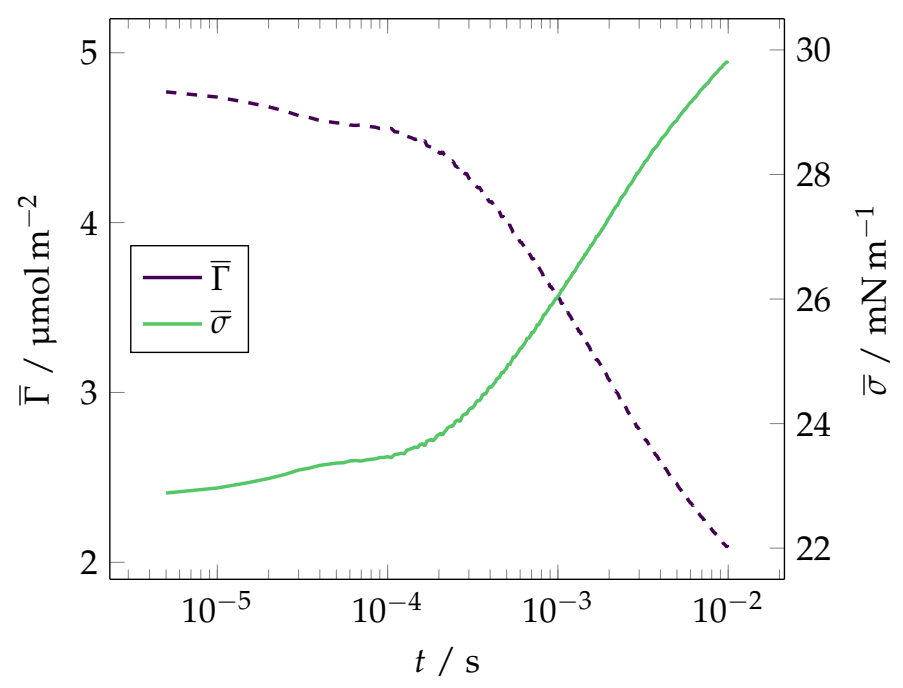

Figure 7. Mean surfactant concentration at the liquid-gas interface and mean surface tension during spreading of a droplet with insoluble surfactant.

\subsection{Spreading with Soluble Surfactant}

In the following, we consider spreading droplets with soluble surfactant with fluid and sorption properties as described in Section 2.3. Here, we also considered varying initial surfactant distributions between bulk and interface. The total amount of surfactant was chosen such that, for the hemispherical droplet of volume $6 \mathrm{pL}$ at equilibrium between bulk and interface concentration, the surface tension is $23 \mathrm{mN} \mathrm{m}^{-1}$, similar to the previously presented simulation for an insoluble surfactant. For the soluble case, this leads us to a surfactant amount of $n=1.164 \times 10^{-14} \mathrm{~mol}$ per droplet. To investigate the influence of the initial surfactant distribution, the ratios of initial surfactant concentrations at the interface to the equilibrium value $\Gamma_{0} / \Gamma_{\text {eq }}=\{0,0.25,0.5,0.75,1\}$ with $\Gamma_{\text {eq }}=4.727 \times 10^{-6} \mathrm{~mol} \mathrm{~m}^{-2}$ were considered. Table 1 shows the corresponding interface and bulk concentrations as well as the initial surface tensions.

Table 1. Surface tension and surfactant concentrations for different initial surfactant distributions.

\begin{tabular}{llccccc}
\hline$\Gamma_{0} / \Gamma_{\text {eq }}$ & & 0 & 0.25 & 0.5 & 0.75 & 1 \\
$\Gamma_{0}$ & in $\mathrm{mol} \mathrm{mm}^{-2}$ & 0 & 1.182 & 2.363 & 3.545 & 4.727 \\
$c_{0}$ & in $\mathrm{mol} \mathrm{m}^{-3}$ & 1.939 & 1.690 & 1.440 & 1.190 & 0.941 \\
$n$ & in $10^{-14} \mathrm{~mol}$ & 1.164 & 1.164 & 1.164 & 1.164 & 1.164 \\
$\sigma(t=0)$ & in $\mathrm{mN} \mathrm{m}^{-1}$ & 35 & 32.09 & 29.12 & 26.09 & 23 \\
\hline
\end{tabular}


Figure 8 shows the wetted diameter over time for varying initial surfactant concentrations at the liquid-gas interface. Additionally, the wetted diameter for the case of an insoluble surfactant presented above is shown. For $\Gamma_{0} / \Gamma_{\text {eq }}=1$, the droplet spreads similarly to the insoluble case. Even after several milliseconds of spreading, the droplet spreads only marginally slower than the droplet in the insoluble surfactant case. With decreasing $\Gamma_{0} / \Gamma_{\mathrm{eq}}$, the droplet spreads faster through the initial stage. For later times $(t \gtrsim 1 \mathrm{~ms})$, however, the droplets with different initial surfactant distributions show a similar spreading behavior.

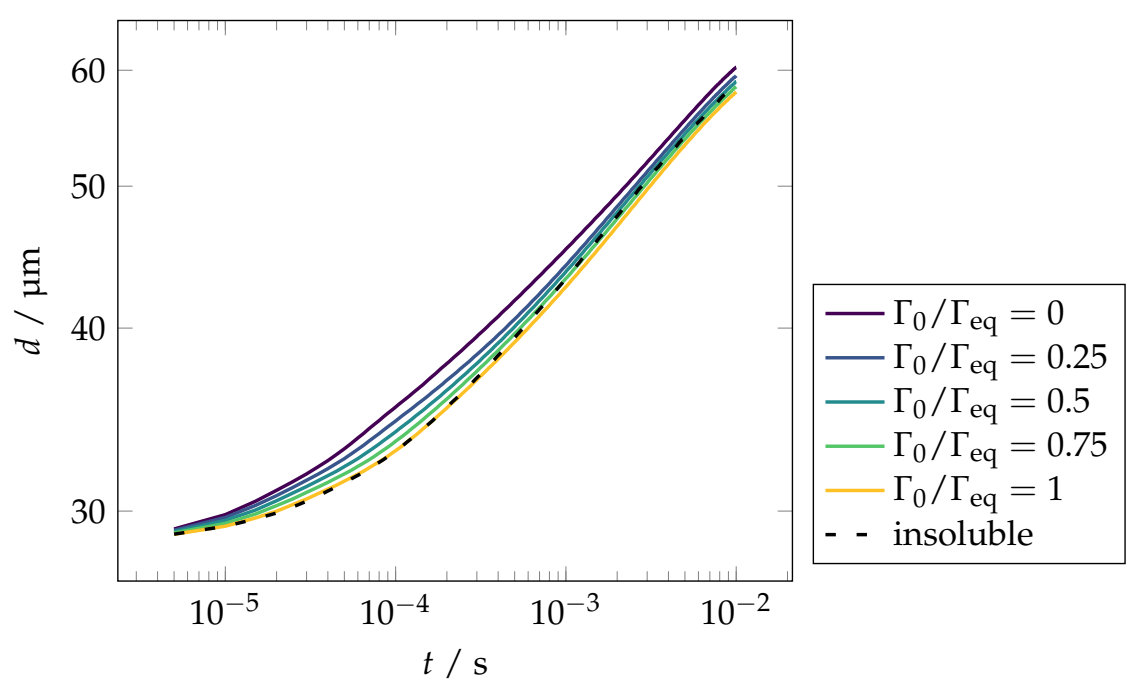

Figure 8. Influence of the initial surfactant concentrations on the wetting behavior.

Figure 9 shows the corresponding mean surface tensions. In agreement with Table 1, the initial surface tension varies with surfactant concentration at the interface. Similar to the wetted diameter data, the case with $\Gamma_{0} / \Gamma_{\text {eq }}=1$ agrees well with the data for the droplet with insoluble surfactant. Even for the soluble surfactant, the surface tension increases over the entire simulated time. However, for $t$ approaching $10 \mathrm{~ms}$, the increasing influence of solubility becomes evident. The surface tension increases slower in the case of soluble surfactants, since additional surfactant is adsorbed at the interface from the bulk of the droplet. Interestingly, all considered cases except the one with an initially surfactant free interface show in the beginning an increase in surface tension while the droplet spreads. While this is clearly visible for the higher initial concentrations, it is barely noticeable in the case of $\Gamma_{0} / \Gamma_{\text {eq }}=0.25$. After reaching a momentary maximum, the surface tension decreases in the cases of $\Gamma_{0} / \Gamma_{\text {eq }}=\{0.25,0.5,0.75\}$. For the initially surfactant free interface, the surface tension decreases monotonically over the simulated timespan, while, in the case of initial equilibrium between bulk and interface concentration, the surface tension increases monotonically. Towards the end of the observed timespan, the difference in mean surface tension between the considered cases has decreased from initially $12 \mathrm{mN} \mathrm{m}^{-1}$ to approximately $2 \mathrm{mN} \mathrm{m}^{-1}$. In all cases, the mean surface tension tends towards the expected equilibrium value $\sigma_{\text {eq }}=28.74 \mathrm{mN} \mathrm{m}^{-1}$ for the given material properties, surfactant amount and droplet volume. 


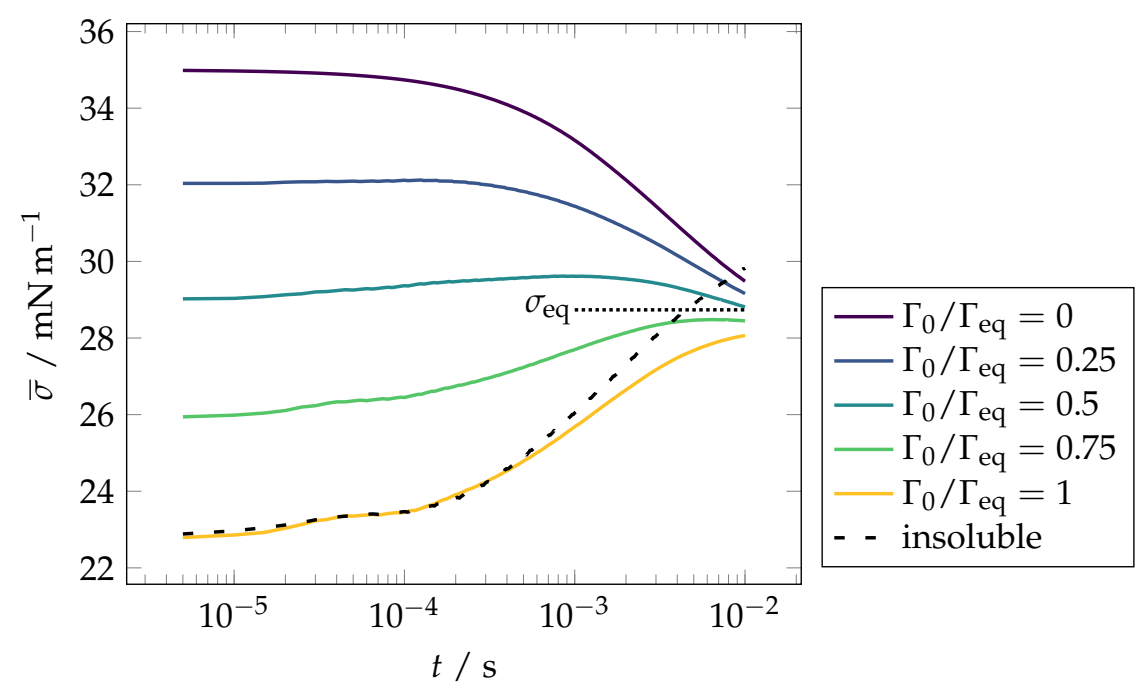

Figure 9. Influence of the initial surfactant concentrations on the mean surface tension. The equilibrium surface tension $\sigma_{\text {eq }}=28.74 \mathrm{mN} \mathrm{m}^{-1}$ as expected for a spherical cap shaped droplet with the given volume and a contact angle of $12^{\circ}$ is indicated by the dotted line.

\section{Discussion}

The results for the spreading droplet with insoluble surfactant show two competing mechanisms that change the wetting behavior compared to a droplet with similar, but constant surface tension. A change in the flow profile due to Marangoni stress at the liquid-gas interface leads in the present case to a slower expansion of the wetted area. The surface active substance hinders flow along the liquid-gas interface towards the three-phase contact line, as this would increase surface tension gradients. This mechanism and the resulting immobilization of the liquid-gas interface during spreading have previously been described also by Jensen and Naire [11], who considered spreading of a viscous droplet with insoluble surfactant over a thin liquid film using lubrication theory. Opposed to this slowing due to the Marangoni effect, the increasing surface tension leads to faster spreading compared to the constant surface tension case. As the droplet spreads, the area of the liquid-gas interface increases. The amount of surfactant present in the droplet, however, remains constant. This leads to a decrease of the surfactant concentration, especially in the case of an insoluble surfactant, and results in an increase of the surface tension. The Kistler dynamic contact angle model employed in the present work relates the dynamic contact angle to the capillary number and predicts with increasing surface tension for a given contact angle also an increasing contact line velocity. Other empirical $[39,40]$ or theoretical $[6,41,42]$ contact angle models predict a similar behavior. Assuming the equilibrium contact angle to remain constant, this leads to the observed, more rapid spreading. For partially wetting systems, such as the one studied here, the equilibrium contact angle would typically be related to the surface tension through Young's equation. However, in the case of complete wetting (i.e., $\theta_{\mathrm{eq}}=0^{\circ}$ ), a constant equilibrium contact angle is a valid assumption. For hydrophilic substrates, such as typical printing substrates, this therefore is a realistic scenario. Moreover, hydrodynamic models for the contact line region including surfactants $([8,9])$ predict even further slowing of the contact line for a given contact angle compared to surfactant free systems due to the Marangoni effect. We expect this to further enhance the effects discussed above. For the droplet with an insoluble surfactant considered here, the competing effects of slowing due to Marangoni flow on the one hand, and a higher contact line velocity due to the increasing surface tension on the other hand cancel each other partly and lead to almost identical wetted diameters on the timescale of a few milliseconds.

Even in the case of a soluble surfactant, as presented in Section 3.4, the increasing interfacial area can result in an increasing surface tension during spreading. Mass balance and geometric arguments in combination with the sorption and surface tension characteristics of the surfactant solution considered 
here alone, i.e., assuming immediate equilibration between bulk and interfacial concentration, lead to an increase of the equilibrium surface tension by $5.74 \mathrm{~m} \mathrm{~N} \mathrm{~m}^{-1}$ from hemispherical shape to a spherical cap with contact angle $12^{\circ}$. Compared to a millimeter-sized drop, the ratio between interfacial area to volume of the droplet is on the order of $100 \times$ larger. Consequently, the amount of surfactant dissolved in the droplets bulk per interfacial area is significantly smaller compared to larger drops with the same bulk and interface surfactant concentrations. Another aspect that should be considered in this context is that a micrometer-sized droplet reaches the same spreading ratio $d / d_{0}$ much faster than a larger, e.g., millimeter-sized, drop. This results in the interfacial area expanding at a higher rate. While the sorption process might be fast compared to wetting for a millimeter-sized drop, for the micrometer-sized droplets adsorption considered here and spreading occur on similar timescales. Even for initial excess surfactant in the bulk, one can observe the surface tension to increase, before decreasing towards the expected equilibrium value $\left(\Gamma_{0} / \Gamma_{\mathrm{eq}}=0.25\right.$ and $\Gamma_{0} / \Gamma_{\mathrm{eq}}=0.5$, Figure 9$)$, demonstrating the competing effects of the expanding interface on the one hand and equilibration of bulk and interface concentration through adsorption on the other hand. For an initially surfactant free interface, the droplet wets most rapidly compared to the other initial surfactant distributions. This is therefore in agreement with the two mechanisms of surfactant decelerated spreading described above. For the surfactant free interface, the surface tension reaches its maximum and, without adsorbed surfactant at the liquid-gas interface, no solutal Marangoni stresses are present, explaining the more rapid expansion of the droplet. With an increasing initial amount of surfactant at the interface, the droplets therefore also spread slower from the beginning. As adsorption starts to equilibrate bulk and interface concentration during the following spreading, the droplets show a more and more similar behavior.

The surfactant, often used as an additive to improve wettability, in our case actually slows spreading. For fast processes such as, e.g., industrial inkjet printing or other coating processes, this might result in smaller wetted areas on the relevant timescales. Somewhat counterintuitively, an initially high surface tension may lead to faster spreading within the capillary-viscous spreading regime. On the other hand, a low surface tension leads to better equilibrium wetting for partially wetting systems. Therefore, the adsorption rate of the surfactant from bulk to the interface and the related dynamic surface tension properties of the system pose an interesting tuning parameter to optimize the wetting process. Due to the competition between adsorption and expansion of the interfacial area, we expect this to be especially relevant to small, micrometer-sized droplets. Moreover, depending on the application, different spreading dynamics might be desirable. A systematic selection of surfactants with specific sorption dynamics might therefore be a way to design or optimize technical processes.

While dynamic surface tension data and sorption model parameters for aqueous systems are available for a large number of surfactants [25,43], only limited data for other solvents are available. Future works should therefore investigate sorption dynamics of non-aqueous systems, such as, e.g., energy curable inks used in a wide range of inkjet applications [32]. Our numerical model might be helpful in finding optimal sorption and surface tension properties for such processes including droplet impact, collision, or coalescence.

\section{Conclusions}

We presented a numerical model for two-phase flows with moving contact lines including an insoluble or soluble surfactant. We briefly presented two verification cases for the sorption model and Marangoni stresses with good to excellent agreement of our model with the corresponding reference solutions. Following that, the model was applied to spreading, micrometer-sized droplets with insoluble and soluble surfactant. In the case of an insoluble surfactant, two competing effects could be identified: (i) immobilization of the interface due to the Marangoni effect and (ii) an increasing surface tension due to the expanding interface and the related decreasing surfactant concentration. Even in the case of a soluble surfactant, these effects could be observed for the present system, while adsorption from the bulk of the droplet could only partly compensate for the increasing area of the liquid-gas interface. 
Supplementary Materials: Data presented in this article are available under the PID: http:/ /hdl.handle.net/ 11304/f278cb72-b0fb-4b34-9eca-a1bb6f840fb5.

Author Contributions: Conceptualization, P.H., B.B., and P.S.; methodology, T.A. and T.G.-R.; software, T.A.; validation, T.A.; investigation, T.A.; writing-original draft preparation, T.A.; writing-review and editing, T.G.-R., P.H., B.B., P.S.; supervision, T.G.-R., P.H., B.B., and P.S.; funding acquisition, P.S.

Funding: This research was funded by Heidelberger Druckmaschinen AG.

Acknowledgments: Calculations for this research were conducted on the Lichtenberg high performance computer of the TU Darmstadt. The first author kindly acknowledges the support by the German Research Foundation (DFG) within the Collaborative Research Centre 1194 "Interaction between Transport and Wetting Processes" as associated member of the CRC.

Conflicts of Interest: The authors declare no conflict of interest. Contributions by the funders are as indicated under "Author Contributions".

\section{References}

1. Derby, B. Inkjet printing of functional and structural materials: Fluid property requirements, feature stability, and resolution. Ann. Rev. Mater. Res. 2010, 40, 395-414. [CrossRef]

2. Morita, N.; Hamazaki, T.; Ishiyama, T.; Motosugi, Y.; Hirakata, S. Breakthroughs required in piezo-on-demand inkjets for production printing: Satellite drops, ink penetration and evaporation. Soc. Imaging Sci. Technol. 2016, 2016, 79-82.

3. Bonn, D.; Eggers, J.; Indekeu, J.; Meunier, J.; Rolley, E. Wetting and spreading. Rev. Mod. Phys. 2009, 81, 739-805. [CrossRef]

4. Brutin, D.; Starov, V. Recent advances in droplet wetting and evaporation. Chem. Soc. Rev. 2018, 47, 558-585. [CrossRef] [PubMed]

5. Harikrishnan, A.; Dhar, P.; Gedupudi, S.; Das, S.K. Effect of interaction of nanoparticles and surfactants on the spreading dynamics of sessile droplets. Langmuir 2017, 33, 12180-12192. [CrossRef] [PubMed]

6. Tanner, L.H. The spreading of silicone oil drops on horizontal surfaces. J. Phys. D Appl. Phys. 1979, 12, $1473-1484$. [CrossRef]

7. Kovalchuk, N.; Matar, O.; Craster, R.; Miller, R.; Starov, V. The effect of adsorption kinetics on the rate of surfactant-enhanced spreading. Soft Matter 2016, 12, 1009-1013. [CrossRef]

8. Cox, R. The dynamics of the spreading of liquids on a solid surface. Part 2. Surfactants. J. Fluid Mech. 1986, 168, 195-220. [CrossRef]

9. Joanny, J.F. Kinetics of spreading of a liquid supporting a surfactant monolayer: Repulsive solid surfaces. J. Colloid Interface Sci. 1989, 128, 407-415. [CrossRef]

10. Clay, M.A.; Miksis, M.J. Effects of surfactant on droplet spreading. Phys. Fluids 2004, 16, 3070-3078. [CrossRef]

11. Jensen, O.; Naire, S. The spreading and stability of a surfactant-laden drop on a prewetted substrate. J. Fluid Mech. 2006, 554, 5-24. [CrossRef]

12. Karapetsas, G.; Chandra Sahu, K.; Matar, O.K. Evaporation of sessile droplets laden with particles and insoluble surfactants. Langmuir 2016, 32, 6871-6881. [CrossRef] [PubMed]

13. Karapetsas, G.; Craster, R.V.; Matar, O.K. On surfactant-enhanced spreading and superspreading of liquid drops on solid surfaces. J. Fluid Mech. 2011, 670, 5-37. [CrossRef]

14. Teigen, K.E.; Song, P.; Lowengrub, J.; Voigt, A. A diffuse-interface method for two-phase flows with soluble surfactants. J. Comput. Phys. 2011, 230, 375-393. [CrossRef] [PubMed]

15. Lakshmanan, P.; Ehrhard, P. Marangoni effect caused by contaminants adsorbed on bubble surfaces. J. Fluid Mech. 2010, 647, 143-161. [CrossRef]

16. Alke, A.; Bothe, D. 3D Numerical Modeling of Soluble Surfactant at Fluidic Interfaces Based on the Volume-of-Fluid Method. Fluid Dyn. Mater. Process. 2009, 5, 345-372.

17. James, A.J.; Lowengrub, J. A surfactant-conserving volume-of-fluid method for interfacial flows with insoluble surfactant. J. Comput. Phys. 2004, 201, 685-722. [CrossRef]

18. Open CFD Ltd. OpenFOAM User Guide Version 1.6; Open CFD Ltd.: London, UK, 2009.

19. Gurumurthy, V.T.; Rettenmaier, D.; Roisman, I.V.; Tropea, C.; Garoff, S. Computations of spontaneous rise of a rivulet in a corner of a vertical square capillary. Colloids Surf. A Physicochem. Eng. Asp. 2018, 544, 118-126. [CrossRef] 
20. Rettenmaier, D. Numerical Simulation of Shear Driven Wetting. Ph.D. Thesis, Technische Universität Darmstadt, Darmstadt, Germany, 2019.

21. Brackbill, J.U.; Kothe, D.B.; Zemach, C. A Continuum Method for Modeling Surface Tension. J. Comput. Phys. 1992, 100, 335-354. [CrossRef]

22. Yokoi, K. A density-scaled continuum surface force model within a balanced force formulation. J. Comput. Phys. 2014, 278, 221-228. [CrossRef]

23. Deising, D.; Marschall, H.; Bothe, D. A unified single-field model framework for Volume-Of-Fluid simulations of interfacial species transfer applied to bubbly flows. Chem. Eng. Sci. 2016, 139, 173-195. [CrossRef]

24. Hardt, S.; Wondra, F. Evaporation model for interfacial flows based on a continuum-field representation of the source terms. J. Comput. Phys. 2008, 227, 5871-5895. [CrossRef]

25. Chang, C.H.; Franses, E.I. Adsorption dynamics of surfactants at the air/water interface: A critical review of mathematical models, data, and mechanisms. Colloids Surf. A Physicochem. Eng. Asp. 1995, 100, 1-45. [CrossRef]

26. Kistler, S.F. Hydrodynamics of Wetting. In Wettability; Berg, J.C., Ed.; Marcel Dekker: New York, NY, USA, 1993.

27. Antritter, T.; Mayer, M.; Hachmann, P.; Wörner, M. Suppressing artificial equilibrium states caused by spurious currents in droplet spreading simulations with dynamic contact angle model. Prog. Comput. Fluid Dyn. 2019. (in press).

28. Issa, R.I. Solution of the implicitly discretised fluid flow equations by operator-splitting. J. Comput. Phys. 1986, 62, 40-65. [CrossRef]

29. Deshpande, S.S.; Anumolu, L.; Trujilo, F. Evaluating the performance of the two-phase flow solver interFoam. Comput. Sci. Discov. 2012, 5, 014016. [CrossRef]

30. Hoath, S.D. Fundamentals of Inkjet Printing: The Science of Inkjet And Droplets; John Wiley \& Sons: Hoboken, NJ, USA, 2016.

31. Graindourze, M. UV-Curable Inkjet Inks and Their Applications in Industrial Inkjet Printing, Including Low-Migration Inks for Food Packaging. In Handbook of Industrial Inkjet Printing: A Full System Approach; Zapka, W., Ed.; Wiley Online Library: Hoboken, NJ, USA, 2018; Volume 1, Chapter 6, pp. 129-150.

32. Bale, M. A System Aproach to Develop New Platforms of Industrial Inkjet Inks. In Handbook of Industrial Inkjet Printing: A Full System Approach, Zapka, W., Ed.; Wiley VCH: Karlsruhe, Germany, 2018; Volume 1, Chapter 3, pp. 23-58.

33. Joos, P.; Serrien, G. Adsorption kinetics of lower alkanols at the air/water interface: effect of structure makers and structure breakers. J. Colloid Interface Sci. 1989, 127, 97-103. [CrossRef]

34. Defay, R.; Hommelen, J.R., III. The importance of diffusion in the adsorption process of some alcohols and acids in dilute aqueous solutions. J. Colloid Sci. 1959, 14, 411-418. [CrossRef]

35. Ward, A.F.H.; Tordai, L. Time-dependence of boundary tensions of solutions I. The role of diffusion in time-effects. J. Chem. Phys. 1946, 14, 453-461. [CrossRef]

36. Li, X.; Shaw, R.; Evans, G.M.; Stevenson, P. A simple numerical solution to the Ward-Tordai equation for the adsorption of non-ionic surfactants. Comput. Chem. Eng. 2010, 34, 146-153. [CrossRef]

37. Ferri, J.K.; Stebe, K.J. Which surfactants reduce surface tension faster? A scaling argument for diffusion-controlled adsorption. Adv. Colloid Interface Sci. 2000, 85, 61-97. [CrossRef]

38. Young, N.O.; Goldstein, J.S.; Block, M.J. The motion of bubbles in a vertical temperature gradient. J. Fluid Mech. 1959, 6, 350-356. [CrossRef]

39. Jiang, T.S.; Soo-Gun, O.; Slattery, J.C. Correlation for dynamic contact angle. J. Colloid Interface Sci. 1979, 69, 74-77. [CrossRef]

40. Bracke, M.; De Voeght, F.; Joos, P. The kinetics of wetting: The dynamic contact angle. In Trends in Colloid and Interface Science III; Springer: New York, NY, USA, 1989; pp. 142-149.

41. Voinov, O. Hydrodynamics of wetting. Fluid Dyn. 1976, 11, 714-721. [CrossRef] 
42. Cox, R.G. The dynamics of the spreading of liquids on a solid surface. Part 1. Viscous flow. J. Fluid Mech. 1986, 168, 169-194. [CrossRef]

43. Miller, R.; Aksenenko, E.; Fainerman, V. Dynamic interfacial tension of surfactant solutions. Adv. Colloid Interface Sci. 2017, 247, 115-129. [CrossRef]

(C) 2019 by the authors. Licensee MDPI, Basel, Switzerland. This article is an open access article distributed under the terms and conditions of the Creative Commons Attribution (CC BY) license (http:/ / creativecommons.org/licenses/by/4.0/). 